\title{
Regulation of tamoxifen sensitivity by a PAK1-EBP1 signalling pathway in breast cancer
}

\author{
A Ghosh ${ }^{1,2}$, S Awasthi ${ }^{1,2}$, J R Peterson ${ }^{3}$ and A W Hamburger ${ }^{\star, 1,2}$ \\ ${ }^{1}$ Greenebaum Cancer Center, University of Maryland School of Medicine, BRB 9-029, 655 W. Baltimore Street, Baltimore, MD \\ 21201, USA; ${ }^{2}$ Department of Pathology, University of Maryland School of Medicine, Baltimore, MD, USA and ${ }^{3}$ Cancer Biology \\ Program, Fox Chase Cancer Center, Philadelphia, PA, USA
}

Background: EBP1, an ErbB3-binding protein, sensitises breast cancer cells to tamoxifen in part by decreasing ErbB2 protein levels. The p21-regulated serine/threonine kinase PAK1, implicated in tamoxifen resistance, phosphorylates EBP1 in vitro and in vivo at T261. Phosphorylation of EBP1 at this site induces tamoxifen resistance. We thus postulated that inhibition of PAK1 activity, by restoring EBP1 function, could ameliorate the hormone refractory phenotype of ErbB2-overexpressing breast cancer cells.

Methods: Effects of EBP1 on ErbB2 levels were measured by western blotting. Effects of EBP1 and IPA-3 on tamoxifen sensitivity were measured using a tetrazolium based cell viability assay.

Results: Transient transfection studies indicated that an EBP1 T261E mutant, which mimics EPB1 phosphorylated by PAK1, increased ErbB2 protein levels. An EBP1 T261A mutant, unable to be phosphorylated by PAK1, ameliorated PAK1-induced tamoxifen resistance, suggesting that phosphorylation of EBP1 by PAK1 contributes to tamoxifen resistance. We then tested if pharmacological inhibition of PAK1 activity might render hormone resistant cells, which endogenously overexpress PAK1, tamoxifen sensitive. IPA-3, a specific small MW PAK1 inhibitor, sensitised cells to tamoxifen only when EBP1 was ectopically expressed. IPA had no effect on tamoxifen resistance in T47D cells in which EBP1 protein had been ablated by shRNA. The IPAinduced increase in tamoxifen sensitivity was accompanied by a decrease in ErbB2 levels only in EBP1-overexpressing cells.

Conclusion: These studies suggest that phosphorylation of EBP1 may be one mechanism of PAK1-induced hormone resistance and that PAK1 inhibitors may be useful in cells in which EBP1 is overexpressed.

Newly diagnosed oestrogen receptor-positive breast cancers are commonly treated with drugs that interrupt oestrogenic stimulation of cell growth: classically tamoxifen and more recently the aromatase inhibitors. However, tamoxifen treatment is limited by the development of resistance and disease relapse (Clarke et al, 2001). Endocrine resistance is often associated with enhanced expression of members of the ErbB receptor family, especially EGFR and ErbB2, and activation of the AKT (Kurokawa et al, 2000) and MAPK (Jelovac et al, 2005) ErbB2/3 downstream signal transduction pathways. A further understanding of the role that ErbB receptors has in development of hormone resistance is important, as approximately half of breast cancers that overexpress ErbB2 also express hormone receptors (PiccartGebhart et al, 2005).

Activation of the ErbB2-stimulated serine/threonine kinase P21-activated kinase 1 (PAK1) leads to the development of tamoxifen resistance. P21-activated kinase 1 is phosphorylated and activated by Rac and AKT in breast cancer cells after treatment with the ErbB3/4 ligand heregulin (HRG) (Vadlamudi et al, 2000). Activation of PAK1 inhibits tamoxifen action in vitro and in animal models (Rayala et al, 2006). P21-activated kinase 1 -induced tamoxifen resistance has been linked to its phosphorylation of $\mathrm{ER} \alpha$ at S305 (Rayala et al, 2006). Clinically, the nuclear localisation of PAK1 is associated with tamoxifen resistance in a subset of

*Correspondence: Dr AW Hamburger; E-mail: ahamburg@som.umaryland.edu

Received 27 September 2012; revised 14 December 2012; accepted 18 December 2012; published online 29 January 2013

(c) 2013 Cancer Research UK. All rights reserved 0007-0920/13 
ER-positive tumours (Holm et al, 2006). P21-activated kinase 1 gene and protein amplification is also a predictor of recurrence and tamoxifen resistance in postmenopausal breast cancer (Bostner et al, 2007, 2010; Kok et al, 2011). P21-activated kinase 1 was recently identified as an oncogene that activates the MAPK pathway and c-MET in breast cancer (Shrestha et al, 2011). Inhibition of PAK1 by shRNA in breast cancer cells results in apoptosis and downregulation of E2F1-regulated genes (Ong et al, 2011).

The biological activity and expression of ErbB2 and ErbB3 are regulated by a host of interacting proteins that may be potential targets for development of new therapies for treatment of hormone resistance. An ErbB3-binding protein (EBP1) was isolated in our laboratory during a yeast two-hybrid screen for ErbB3-interacting proteins (Yoo et al, 2000). Overexpression of EBP1 inhibits growth of ErbB2/3 expressing breast cancer cell lines, promotes G2/M cell cycle arrest and cellular differentiation (Lessor et al, 2000), and inhibits AKT activation and cell proliferation in response to HRG (Zhang et al, 2008). The biological activity of EBP1 is regulated by the kinases $\mathrm{PKC} \delta$ (Ahn et al, 2006; Liu et al, 2006) and PAK1 (Akinmade et al, 2008). Phosphorylation of EBP1 at S360 by PKC $\delta$ (Ahn et al, 2006) and T261 by PAK1 (Akinmade et al, 2008) has been demonstrated in vitro and in vivo (Akinmade et al, 2008).

Recent work from our laboratory indicates a role for EBP1 in modulating hormone sensitivity. Overexpression of EBP1 results in an increase in tamoxifen sensitivity in hormone sensitive cells (Akinmade et al, 2008) and a decrease in ErbB2 protein levels (Zhang et al, 2008). Silencing of EBP1 results in the increased expression of ErbB2 in tamoxifen sensitive MCF-7 (Zhang et al, 2008) and T47D cells ( $\mathrm{Lu}$ et al, 2011), and the development of tamoxifen resistance. This tamoxifen resistance is overcome by HER-2 kinase inhibitors ( $\mathrm{Lu}$ et al, 2011), suggesting that EBP1's effect on tamoxifen sensitivity may be mediated by its ability to modulate ErbB2 expression. Transfection of an EBP1 mutant, which mimics PAK1-induced phosphorylation at T261 induces tamoxifen resistance in MCF-7 cells.

As our previous work only examined hormone sensitive cells, we sought to determine if PAK1's interactions with EBP1 might affect ErbB2 levels and the response to tamoxifen in hormone resistant cells. We found that a T261E EBP1 PAK1 phosphomimetic mutant increased ErbB2 levels. An EBP1 T261A mutant that was unable to be phosphorylated by PAK1 reversed PAK1-induced tamoxifen resistance. Pharmacological reduction of PAK1 activity by IPA-3 in hormone resistant LTLT-Ca cells, in which both PAK1 and ErbB2 are endogenously overexpressed, inhibited cell growth, but did not induce tamoxifen sensitivity. However, IPA-3 sensitised LTLT-Ca cells to tamoxifen when EBP1 was overexpressed. IPA-3 decreased ErbB2 levels only when EBP1 was overexpressed. These studies suggest that phosphorylation of EBP1 may be one mechanism of PAK1-induced hormone resistance and that PAK1 inhibitors may be useful in cells in which EBP1 is overexpressed.

\section{MATERIALS AND METHODS}

Cell culture. MCF-7 and AU565 cells were obtained from the American Type Culture Collection (Manassas, VA, USA). T47D cells were a gift of Dr Stuart Martin, University of Maryland School of Medicine. All cell lines were maintained at $37^{\circ} \mathrm{C}$ in a humidified atmosphere of $5 \% \mathrm{CO}_{2}$ in air in RPMI 1640 (Biofluids, Rockville, MD, USA) and 10\% FBS (Sigma, St Louis, MO, USA). LTLT-Ca cells were a gift of Dr Angela Brodie, University of Maryland School of Medicine and maintained as described (Jelovac et al, 2005). LTLT-Ca cells were derived from aromatase-transfected MCF-7 cells made tamoxifen resistant by passage in vivo in the presence of letrozole (Sabnis et al, 2009).
Reagents. Heregulin $\beta 1$ (HRG $\beta 1$ ) was obtained from R \& D Systems Inc. (Minneapolis, MN, USA), 4-hydroxy-tamoxifen $(\mathrm{OHT})$ and $17 \beta$-estradiol from Sigma.

Plasmids. A full-length EBP1 cDNA (GenBank NM006191) was generated by PCR with specific reverse and forward primers containing XhoI and BamHI restriction sites using a pcDNA-EBP1 vector as a template (Xia et al, 2001a). This cDNA includes all three possible translation initiation sites of EBP1 and encodes the largest form of the protein (Xia et al, 2001b). The hygromycin resistant wild-type EBP1 plasmid was constructed by cloning this full-length EBP1 into the XhoI and BamH1 sites of the pAGFP1-Hyg-C1 vector (Clontech, Palo Alto CA, USA). The EBP1 T261A expression plasmid was constructed in pcDNA3 Hygro (Invitrogen, Carlsbad, CA, USA) with a GFP tag. The orientation and integrity of cDNA inserts in the newly constructed plasmids were confirmed by automated DNA sequencing in the core laboratory of the University of Maryland School of Medicine. Constitutively active PAK1 (T423E) cloned into pcDNA3 was a gift of Dr Z Luo (Zang et al, 2002).

Creation of stably transfected cell lines. To establish LTLT-Caoverexpressing EBP1 stable transfectants, subconfluent cells in 100$\mathrm{mm}$ tissue culture dishes were transfected with $10 \mu \mathrm{g}$ of pAGFP1Hyg-C1 or pAGFP1-Hyg-C1-EBP1 or pcDNA-GFP-Hyg or pcDNA-GFP-Hyg-EBP1 T261A expression plasmids using Fugene6 (Roche, Indianapolis, IN, USA) according to the manufacturer's protocol. Cells were selected in hygromycin $\left(20 \mu \mathrm{g} \mathrm{ml}^{-1}\right)$ for 4 weeks and mass cultures obtained. Cells were further selected for high GFP-EBP1 expression by FACS sorting. MCF-7 cells stably expressing a constitutively active PAK1 (T423E) were made by transfecting cells as described above with the pcDNA3 vector expressing PAK1 T423E. Cells were selected in $500 \mu \mathrm{g} \mathrm{ml}^{-1}$ of G418. The T47D EBP1-silenced cell line and the BT474-EBP1-overexpressing cell line have been previously described (Lu et al, 2011).

Western blot assay. Total cell lysates were prepared by direct lysis with HNTG buffer (50 mM HEPES pH 7.5, $150 \mathrm{~mm} \mathrm{NaCl}, 1.5 \mathrm{~mm}$ $\mathrm{MgCl}_{2}, 0.01 \%$ Triton X100, $10 \%$ glycerol). Protein concentrations were determined using the Bio-Rad detergent compatible protein assay kit (Bio-Rad, Hercules, CA, USA). The samples were mixed with Nupage sample buffer and resolved by denaturing NuPAGE. Proteins were transferred to PVDF membranes and immunoblotted with the appropriate primary and secondary antibodies. An ECL detection kit (Pierce, Rockford, IL, USA) was used to visualise the bands. Where indicated, images were quantified using IMAGEJ software (NIH, Bethesda, MD, USA).

Antibodies. Primary antibodies included those directed against EBP1 (rabbit, Millipore, Billerica, MA, USA), GFP (mouse, Clontech), GAPDH, p-threonine, PAK1 and phospho-PAK (T423) (rabbit, Cell Signaling, Danvers, MA, USA), tubulin and Actin (rabbit, Sigma). Secondary antibodies included goat anti-rabbit HRP (Bio-Rad) and sheep anti-mouse HRP (Amersham, Piscataway NJ, USA).

Immunoprecipitation. EBP1 was immunoprecipitated from MCF-7 and LTLT-Ca cell lysates using anti-EBP1 antibody as previously described (Akinmade et al, 2008). The immunoprecipitated proteins were resolved by SDS-PAGE and analysed by western blotting using anti phospho-threonine (Cell Signaling) or EBP1 primary antibodies and a peroxidase-conjugated mouse antirabbit conformation-specific secondary antibody (Cell Signaling).

Cell growth assays. For studies assessing the effect of tamoxifen on cell growth, cells $\left(2 \times 10^{3}\right)$ were plated in 96-well plates in complete media. After a 24-hour attachment period, the medium was replaced with medium containing $2 \%$ FBS and $10^{-8} \mathrm{M} 17-\beta$ estradiol and OHT with or without IPA-3 at the indicated concentrations. Cells were refed at day 4 . Relative live cell numbers were determined at day 7 using a Promega proliferation reagent (Promega, Madison, WI, USA) as per the manufacturer's 
instructions with absorbance being read at $490 \mathrm{~nm}$ using a Thermo Multiskan Ascent plate reader (Thermo Scientific, Hudson, NH, USA).

Statistical analysis. Data were analysed using a two-tailed Student's $t$-test. Differences with a $P<0.05$ were deemed significant.

\section{RESULTS}

Effect of an EBP1 T261E mutant on ErbB2 levels. We previously demonstrated that PAK1 phosphorylated EBP1 in vitro at T261 and that a phosphomimetic T261E mutant induced tamoxifen resistance in hormone sensitive MCF-7 cells (Akinmade et al, 2008). As the ability of EBP1 to decrease ErbB2 levels has a role in its induction of tamoxifen sensitivity (Lu et al, 2011), we tested the effect of an EBP1 T261E phosphomimetic on ErbB2 levels. We used AU565 cells, which exhibit a large decrease in ErbB2 levels after ectopic expression of EBP1 (Zhang et al, 2008) (Lu et al, 2011). Transient transfection of wild-type EBP1 decreased ErbB2 protein as expected. However, a phosphomimetic T261E EBP1 increased ErbB2 levels (Figure1). We (Akinmade et al, 2008) and others (Squatrito et al, 2006) have demonstrated the subcellular localisation of the GFP-tagged proteins is identical to that of the endogenous protein. The GFP-EBP1 fusion proteins were expressed at approximately equal levels (Figure 1). This finding suggests that phosphorylation of the T261 site abrogates the ability of EBP1 to decrease ErbB2 protein levels.

An EBP1 T261A mutant reduces PAK1-induced tamoxifen resistance. PAK1 has been demonstrated to induce tamoxifen resistance via phosphorylation of ER $\alpha$ (Rayala et al, 2006). We postulated that EBP1 phosphorylation by PAK1 and the subsequent inhibition of EBP1's ability to reduce ErbB2 levels might also have a role in tamoxifen resistance. We, therefore, tested if an EBP1 T261A mutant that could not be phosphorylated by PAK1 could overcome PAK1-induced tamoxifen resistance. We created MCF-7 cells stably transfected with a constitutively activated PAK1
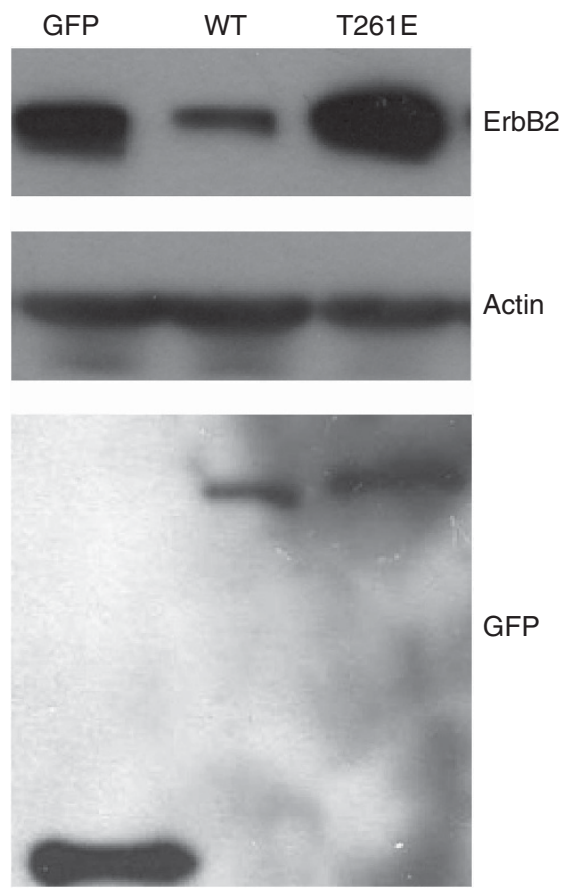

Figure 1. Effect of transfection of mutant EBP1 on ErbB2 protein levels. AU565 cells were transiently transfected with wild-type (WT) or mutant (T261E) EBP1. Two days later, lysates were collected and analysed by western blotting with antibodies to ErbB2, actin or GFP as indicated. Data are representative of three experiments.
T423E (Figure 2A). PAK1 levels were increased in these cells as expected. In addition, ErbB2 levels were increased (Figure 2A). We have previously published that EBP1 Thr phosphorylation is increased in MCF-7 cells transfected with PAK1 (Akinmade et al, 2008). As expected, overexpression of a constitutively active $P A K 1$ resulted in tamoxifen resistance (Figure $2 \mathrm{~B}$ ). We next transiently transfected these tamoxifen resistant PAK1 T423E MCF-7 stable transfectants with GFP vector control, wild-type EBP1, T261A or T261E EBP1 mutants and assessed tamoxifen sensitivity. Transfection of the EBP1 T261A mutant was able to partially overcome PAK1-mediated tamoxifen resistance. In keeping with previous data using parental MCF-7 cells (Akinmade et al, 2008), transfection of a wild-type EBP1 increased tamoxifen sensitivity, but not as much as the T261A mutant. The EBP1 T261E mutant increased resistance at low concentrations of tamoxifen (Figure 2C). Equal expression levels of the EBP1 fusion proteins were observed (Figure 2C).

P21-activated kinase 1 inhibitors reverse tamoxifen resistance only when $E B P 1$ is overexpressed. We next tested the hypothesis that PAK1 inhibitors, by reducing PAK1 phosphorylation of EBP1, could reverse tamoxifen resistance. We used the LTLT-Ca cell line, made hormone resistant by in vivo passage in the presence of letrozole. This cell line endogenously overexpresses ErbB2 (Jelovac et al, 2005). We found that this cell line also overexpresses PAK1 as compared with parental MCF-7 cells and that PAK1 is constitutively phosphorylated (Figure $3 \mathrm{~A}$ ). In keeping with the fact that PAK1 is overexpressed, basal threonine phosphorylation of EBP1 was observed in LTLT-Ca cells. No specific threonine phosphorylation of EBP1 was observed in MCF-7 parental cells (Figure 3B) as previously reported (Akinmade et al, 2008).

We tested the effects of IPA-3, a PAK1 inhibitor that interferes with PAK1 activation by altering PAK conformation and potentially blocking opening of the autoinibited state (Deacon et al, 2008; Viaud and Peterson, 2009), on the tamoxifen sensitivity of this line. As little is known about the effects of IPA-3 on proliferation of hormone resistant breast cancer cells, we first tested the response LTLT-Ca cells to IPA-3. Dose-response curves indicated that $3 \mu \mathrm{M}$ IPA-3 inhibited cell proliferation by $\sim 20 \%$ (Figure 3C). As we were interested in using IPA-3 at a $20 \%$ growthinhibitory dose for further combination studies with tamoxifen, we examined its effect on PAK activation at this dose. IPA-3 reduced HRG-induced phosphorylation in serum-starved cells at this concentration by $\sim 50 \%$ as indicated by densitometric analysis of western blots (Figure 3D). Higher non-toxic concentrations (5 and $10 \mu \mathrm{M})$ did not further inhibit phosphorylation under these conditions (data not shown). We next tested the effects of a combination of OHT and IPA-3 on cell growth. As has been reported (Shou et al, 2004; Sabnis et al, 2009), OHT stimulated the growth of the hormone resistant cell line (Figure 3E). The tamoxifen-induced growth increase was abrogated by treatment with IPA-3. However, cells did not become sensitive to tamoxifen after IPA-3 treatment (Figure 3E). Similar results were observed using IC50 doses of IPA (data not shown).

As we demonstrated (Figure 2) that overexpression of wild-type and T261A mutated EBP1 ameliorated PAK1-induced tamoxifen resistance, we hypothesised that IPA-3 might be more effective in LTLT-Ca cells when EBP1 was overexpressed. We reasoned that endogenous PAK1 would be unable to phosphorylate and inactivate a larger pool of EBP1. Thus, inhibition of PAK1 in the presence of high levels of EBP1 might increase active EBP1 levels to a threshold needed to observe EBP1-induced tamoxifen sensitization. Overexpression of EBP1 had no effect on total PAK levels in LTLT-Ca cells. Surprisingly both basal and HRG-stimulated pPAK levels were elevated in EBP1 transfectants (Figure 4A). LTLT-CaEBP1 cells were approximately as sensitive to IPA-3 as vector LTLT-Ca controls (data not shown). Overexpression of EBP1 
A

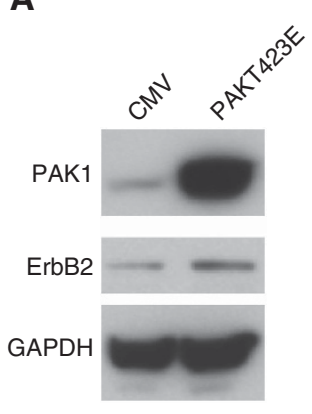

B

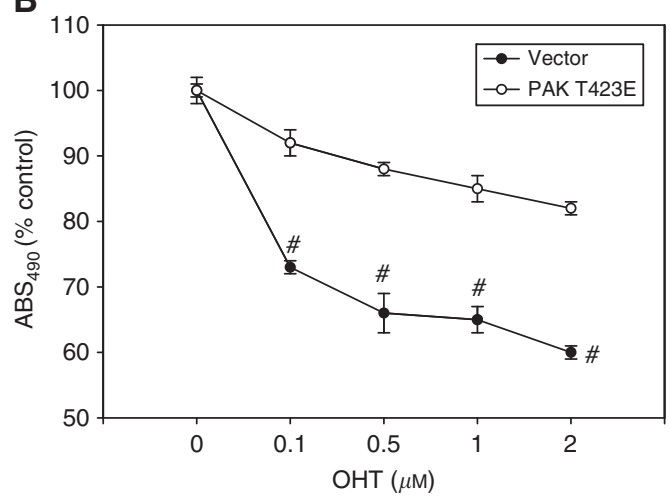

GFP WT E A
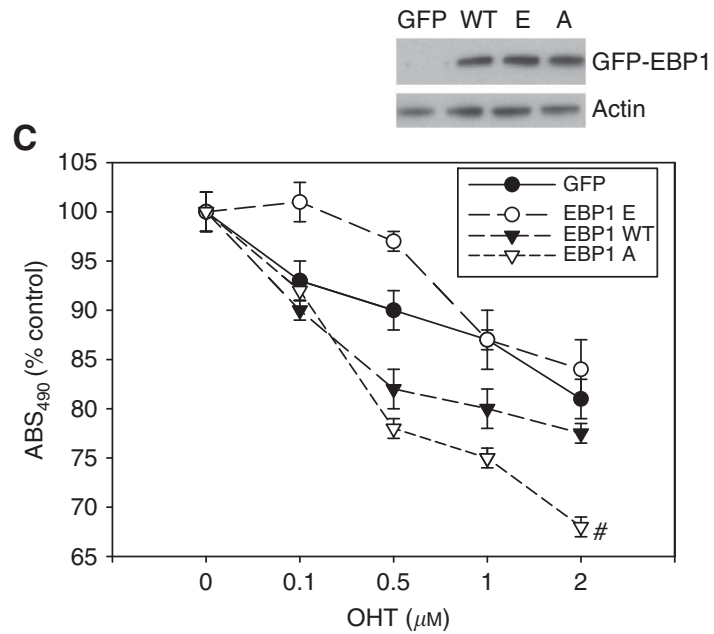

Figure 2. An EBP1 T261A mutant can reduce PAK1-induced tamoxifen resistance. (A) MCF-7 cells were stably transfected with a constitutively active (T423E) PAK1 vector. Cells were lysed and analysed by western blotting for expression of PAK1, ErbB2 or GAPDH as indicated. (B) Tamoxifen sensitivity of MCF-7 cells transfected with constitutively activated PAK1. MCF-7 cells stably transfected with a PAK1 T423E expression vector or a vector control were incubated for 7 days in the presence of $\mathrm{OHT}$ at the indicated concentrations or vehicle control as described in the Materials and Methods. Viable cells were quantified using a Promega cell proliferation assay. Each data point represents the mean \pm s.d. of six wells. Similar results were observed in two independent experiments. ${ }^{\#} P<0.01$ vector controls vs PAK1-transfected cells. (C) Sensitivity of tamoxifen resistant MCF-7 PAK1 T423E cells transfected with EBP1 mutants to tamoxifen. MCF-7 cells stably transfected with a PAK T423E expression vector (see Figure 2B) were transiently transfected with GFP, GFP-WT-EBP1, GFP-EBP1 T261A or GFP-EBP1 T261E in complete media. Sixteen hours after transfection, cells were refed with media with the indicated concentrations of OHT. Growth was assessed 3 days later using a Promega cell proliferation assay as described in the Materials and Methods. Each data point represents the mean \pm s.d. of six wells. Similar results were observed in two independent experiments. ${ }^{\#} P<0.01$ EBP1 T261A-transfected cells vs GFP-transfected cells at $2 \mu \mathrm{m}$ OHT. Expression levels of the $80 \mathrm{kDa}$ EBP1-GFP fusion proteins were measured by western blotting using a GFP antibody (inset).

A
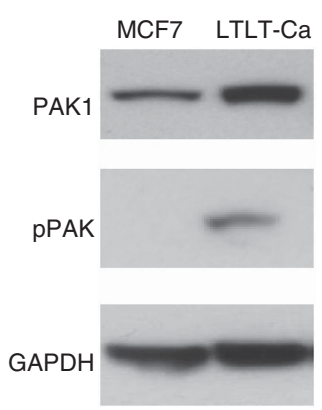

\section{B}

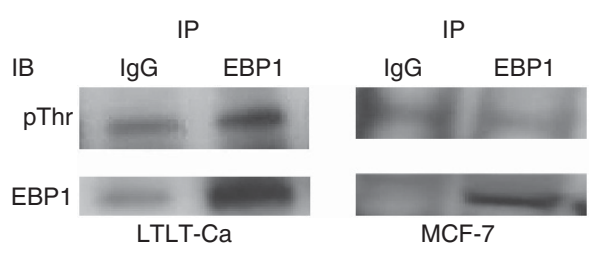

C

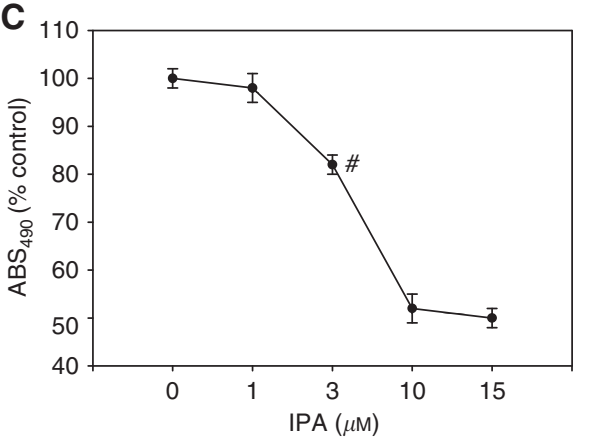

D

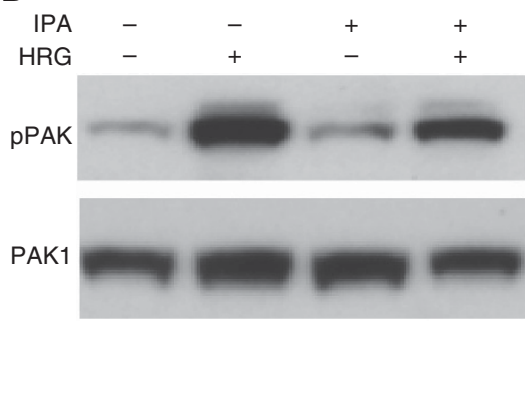

E

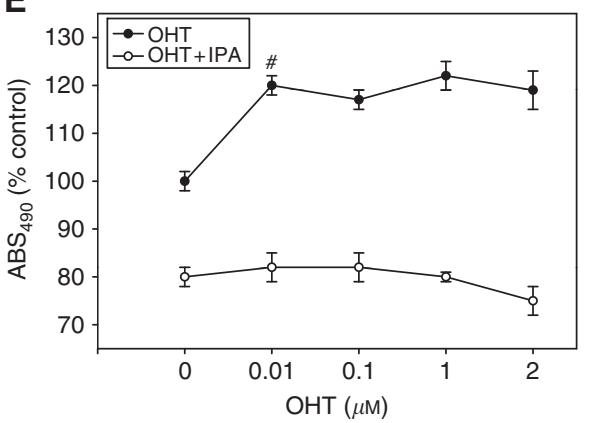

Figure 3. A PAK inhibitor prevents tamoxifen-induced growth of PAK1-overexpressing hormone resistant cells. (A) Hormone resistant cells overexpress PAK1. Lysates of logarithmically growing parental MCF-7 and LTLT-Ca cells were analysed by western blotting for total PAK1, pPAK or GAPDH as indicated. (B) Threonine phosphorylation of EBP1 in LTLT-Ca and MCF-7 cells. Lysates of logarithmically growing LTLT-Ca or MCF-7 cells were immunoprecipitated with either control lgG or EBP1 antibody before separation of proteins by SDS-PAGE. Proteins were transferred to PVDF membranes, and immunoblotted with antibodies to phospho threonine (pThr) or EBP1 as indicated. (C) LTLT-Ca cells were treated with IPA-3 at the indicated concentrations and cell number determined 7 days later as described in the Materials and Methods. ${ }^{\#} P<0.01$ compared with untreated control. (D) Effect of IPA-3 on PAK phosphorylation. Cells were serum starved overnight in media containing $0.5 \%$ FBS with or without $3 \mu \mathrm{M}$ IPA-3 as indicated. Cells were then treated with $50 \mathrm{ng} \mathrm{ml}^{-1}$ of HRG for $60 \mathrm{~min}$. Cell lysates were collected and analysed by western blotting for phospho PAK1 and total PAK1 as indicated. (E) LTLT-Ca cells were treated with the indicated concentrations of OHT with or without IPA-3 $(3 \mu \mathrm{M})$ and cell number determined at day 7 . Representative of five experiments. ${ }^{\#} P<0.01$ compared with untreated control. 
sensitised LTLT-Ca cells to tamoxifen (Figure 4B compared with Figure 3E) and IPA-3 further enhanced sensitivity to tamoxifen. This sensitization was most markedly observed at lower concentrations of tamoxifen. Growth of EBP1 wild-type transfectants was inhibited $20 \%$ at the highest doses of tamoxifen used compared with controls, but $38 \%$ in the presence of IPA as compared with the IPA-3 only treated control. This difference in response between OHT only and OHT-IPA-3-treated groups is statistically significant at $P<0.05$. In addition, growth of cells in the presence of the highest concentration of OHT and IPA was inhibited $48 \%$ compared with untreated controls. These data indicate that overexpression of EBP1 in combination with partial inhibition of PAK1 activity in LTLT-Ca cells were more effective than either condition alone in sensitising cells to tamoxifen (Figure 4B).

We were further interested in determining if the effects of IPA-3 on tamoxifen sensitivity might be mediated by PAK1 phosphorylation of EBP1. We, therefore, created LTLT-Ca cells overexpressing EBP1 T261A, which cannot be phosphorylated by PAK1. Initial growth curves indicated that IPA-3 affected cell growth in approximately the same manner as in LTLC-Ca EBP1 wild-type transfectants (data not shown). EBP1 T261A-transfected cells were more sensitive to tamoxifen at low concentrations than EBP1 wild-type transfectants ( compare Figure 4B and C) similar to results in Figure 2. However, IPA-3 did not significantly affect the sensitivity of EBP1 T261A-transfected cells to tamoxifen as compared with cells treated only with tamoxifen. For example, growth of LTLT-Ca-EBP1 T261A cells was inhibited $20 \%$ at the highest concentration of tamoxifen used and $23 \%$ in the presence of IPA-3 compared with IPA only treated cells. This finding suggests that the ability of IPA-3 to sensitise cells to tamoxifen in EBP1-overexpressing cells was due in part to its ability to prevent EBP1 phosphorylation. Finally, IPA-3 had no effect on tamoxifen sensitivity in hormone resistant T47D cells in which EBP1 protein expression has been silenced and ErbB2 expression increased (Lu et al, 2011) (Figure 4D).

A
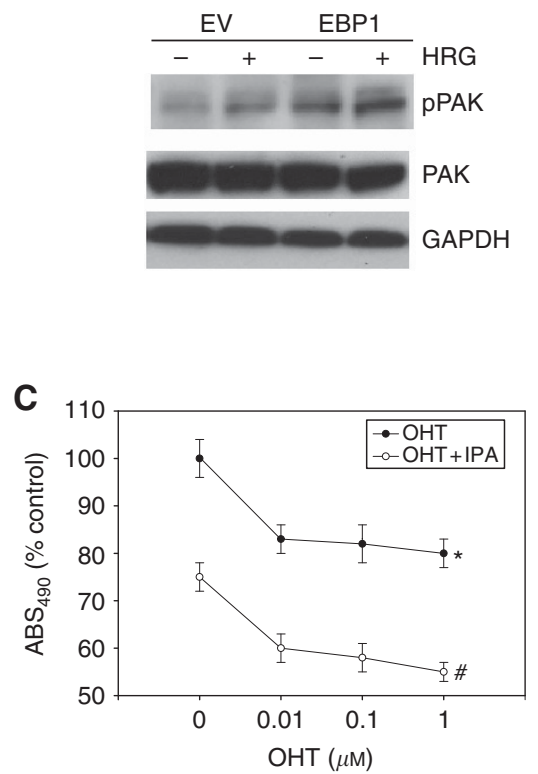

As we previously demonstrated that the induction of sensitivity to tamoxifen by EBP1 was related to its effects on ErbB2 levels, we examined ErbB2 levels in both LTLT-Ca vector controls and EBP1 transfectants. As expected, overexpression of both wild-type and T261A EBP1 decreased ErbB2 levels in LTLT-Ca cells (Figure 5A) consistent with the observation that overexpression of EBP1 sensitised cells to tamoxifen. We next tested the effect of IPA-3 on ErbB2 expression levels in vector control and EBP1 (wild-type and T261A)-overexpressing cells. IPA-3 decreased ErbB2 levels in both wild-type and mutant EBP1-overexpressing cells, but not vector controls (Figure 5B). IPA-3 was able to inhibit basal phosphorylation of MAPK observed in control LTLT-Ca cells (Jelovac et al, 2005) and EBP1-transfected cells in keeping with previously published data that overexpression of PAK1 activates MAPK signalling pathways (Shrestha et al, 2011).

\section{DISCUSSION}

The clinical importance of ErbB heterodimers and their interacting partners in breast cancer development and endocrine resistance has been well-documented (Hynes and Lane, 2005). However, the role of ErbB-binding proteins in hormone resistance has not been as extensively studied. We have previously shown that the ErbB3binding protein EBP1 decreases ErbB2 levels and enhances tamoxifen sensitivity of hormone sensitive cells (Akinmade et al, 2008; Lu et al, 2011). Silencing of EBP1 expression results in increases in ErbB2 levels and development of hormone resistance (Lu et al, 2011). EBP1 is a PAK1 substrate and an EBP1 T261E mutant, that mimics EBP1 phosphorylated by PAK1, induces tamoxifen resistance in hormone sensitive MCF-7 cells (Akinmade et al, 2008). We thus postulated that inhibition of PAK1 activity, by restoring EBP1 function, could ameliorate the hormone refractory phenotype of ErbB2-overexpressing breast cancer cells.
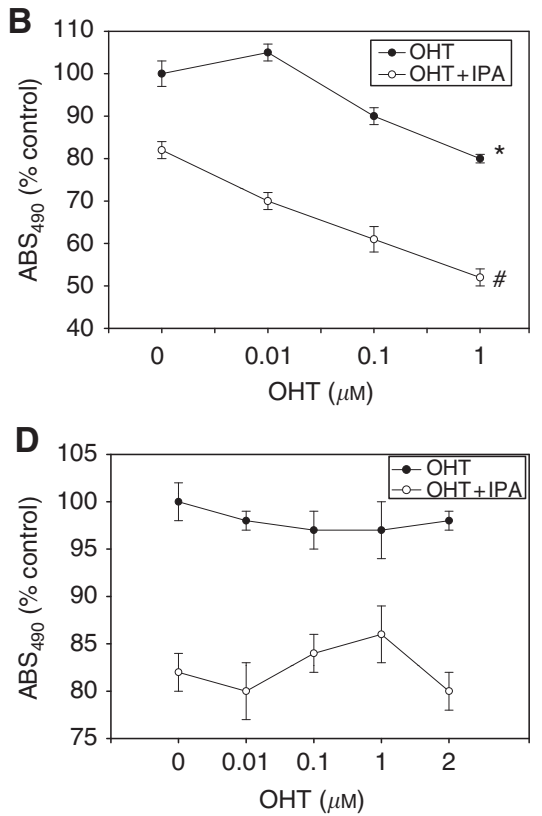

Figure 4. IPA-3 reverses tamoxifen resistance when EBP1 is overexpressed. Expression of pPAK and PAK in control and EBP1-transfected LTLT-Ca cells. (A) LTLT-Ca vector control (EV) or EBP1-transfected cells were serum-starved overnight and treated with $50 \mathrm{ng} \mathrm{ml}^{-1}$ of HRG for $60 \mathrm{~min}$. Cell lysates were analysed by western blotting for PPAK or total PAK1 as indicated. (B, C) LTLT-Ca cells stably transfected with wild-type EBP1 (B) or EBP1 T2621A (C) were treated with the indicated concentrations of OHT with or without IPA-3 $(3 \mu \mathrm{M})$ and cell number determined at day 7 as described in the Materials and Methods. Representative of five experiments ${ }^{\star} P<0.05$ compared with untreated controls, ${ }^{\#} P<0.01$ compared with IPA only treated controls. (D) T47D EBP1-silenced cells were treated with the indicated concentrations of OHT with or without IPA-3 $(3 \mu \mathrm{M})$ and cell number determined at day 7 as described in the Materials and Methods. Representative of two experiments. 
A
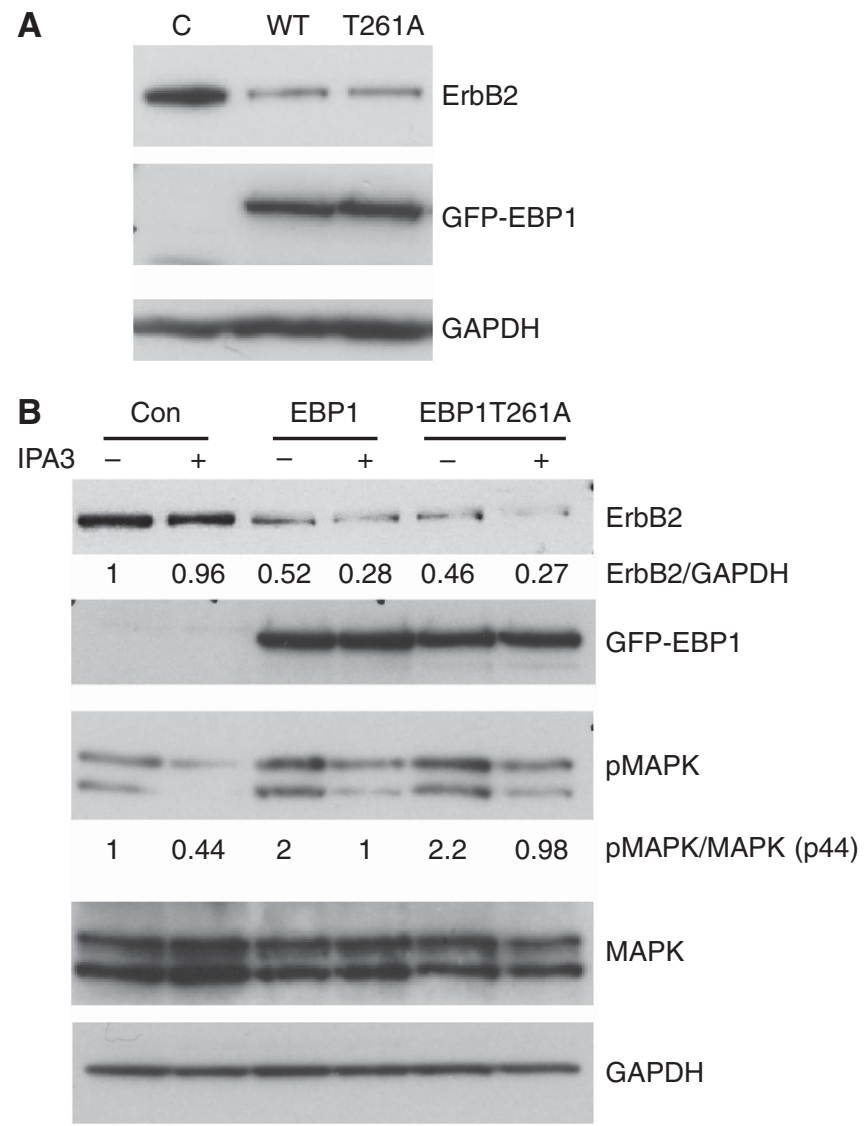

Figure 5. Regulation of ErbB2 levels in LTLT-Ca cells by EBP1 overexpression and IPA-3. (A) ErbB2 expression levels were measured by western blot analysis of logarithmically growing vector control, EBP1 wild-type or T261A-transfected LTLT-Ca cells. Expression of the GFPEBP1 fusion protein and GAPDH was also analysed. (B) LTLT-Ca vector control or wild-type or mutant EBP1-transfected cells were serumstarved overnight in media containing $0.5 \% \mathrm{FBS}$ with or without $3 \mu \mathrm{M}$ IPA-3 as indicated. Cell lysates were collected and analysed by western blotting for ErbB2, GFP-EBP1, PMAPK, MAPK and GAPDH levels as indicated. The numbers below the ErbB2 western blot represent relative densities normalised to GAPDH. The numbers below the PMAPK blot indicate the relative densities of PMAPK normalised to MAPK (p44).

As EBP1's effect on tamoxifen sensitivity is mediated in part by its ability to modulate ErbB2 expression, we tested if the T261E EBP1 phosphomimetic mutant could still decrease ErbB2 levels. In keeping with data indicating that this mutant induces tamoxifen resistance (Akinmade et al, 2008), we found that the phosphomimetic increased ErbB2 protein levels. We postulated that PAK1induced phosphorylation of EBP1 might represent a previously unrecognised mechanism of PAK1-induced tamoxifen resistance. We, therefore, tested if an EBP1 T261A mutant that could not be phosphorylated by PAK1 could overcome PAK1-induced tamoxifen resistance. We found that transfection of the EBP1 T261A mutant could partially reverse PAK-induced tamoxifen resistance. We suggest that phosphorylation of EBP1 may be a novel mechanism of PAK-induced tamoxifen resistance.

We were further interested in determining if inhibition of PAK1 activity could affect sensitivity of hormone resistant cells to tamoxifen, especially as we found for the first time that hormone resistant cells endogenously overexpress PAK1. Results of our proliferation assays indicated that treatment with IPA-3, at a dose which partially inhibits PAK1 phosphorylation, did not sensitise hormone resistant cells to tamoxifen, but did abrogate tamoxifen-induced growth stimulation. The ability of IPA-3 to reverse tamoxifen-induced growth could be due to several factors. PAK1 phosphorylates a large number of substrates that are related to cell growth (Aurora Kinase A, PCBP-1) and apoptosis (Bad, DLC, FKHR) (Dummler et al, 2009; Molli et al, 2009), but not specifically endocrine resistance. P21-activated kinase 1 has also been recently demonstrated to be an oncogene in breast cancer via its activation of both MAPK and MET signalling (Shrestha et al, 2011). It is also possible that complete inhibition of PAK1 activity is needed to observe IPA-3's effects on hormone resistance. However, we were unable to completely inhibit PAK1 phosphorylation at doses of IPA-3 that were not non-specifically toxic. This may be due to the fact that a pool of PAK1 is constitutively activated in LTLT-Ca cells and thus would not be subject to inhibition by IPA-3, which acts by blocking opening of autoinhibited PAK1 dimers.

In contrast, IPA-3 sensitised LTLT-Ca cells to tamoxifen when EBP1 was overexpressed. We observed similar results in the partially hormone resistant BT474 cell line, which has been shown to have high levels of PAK1 (Ong et al, 2011). This cell line can be sensitised to tamoxifen by EBP1 overexpresssion. IPA enhances tamoxifen sensitivity in BT474-EBP1-overexpressing cells, but not in vector controls cells (Supplementary Figure 1). IPA-3 failed to affect hormone resistance of cells in which EBP1 expression had been silenced. The fact that IPA-3 has no effect on tamoxifen sensitivity in the absence of EBP1 or in the presence of endogenous levels of EBP1 suggests that EBP1 had a role in IPA-3-induced sensitization to tamoxifen in EBP1-overexpressing cells. It is possible that the overexpression of $E B P 1$ provides a large pool of protein that cannot be completely phosphorylated by endogenous PAK1. The reduction of PAK1 activity by IPA-3 may increase the pool of nonphosphorylated EBP1 to a threshold that is needed for its biological activity. In the absence of EBP1 overexpression, IPA-3 inhibition of PAK1 activity is insufficient to allow the pool of non-phosphorylated EBP1 to reach this critical level. The fact that the IPA-3induced increase in tamoxifen sensitivity was not observed when the EBP1 T261A mutant was transfected suggests that part of the effect of IPA-3 in the presence of high levels of EBP1 was due to inhibition of PAK1-induced phosphorylation of wild-type overexpressed EBP1. The observation that a PAK1 inhibitor may alter sensitivity to tamoxifen in the presence of high levels of EBP1 may have clinical significance as many localised breast cancers overexpress EBP1 (Ou et al, 2006). We are developing a phospho antibody to the EBP1 T261 site for immunohistochemical analysis of clinical breast cancer samples to establish if there is a correlation between PAK1, phospho EBP1 levels and tamoxifen resistance. Such studies are critical to testing our hypothesis that coexpression of PAK1 and phospho EBP1 is associated with tamoxifen resistance in patients.

The molecular downstream events that led to induction of tamoxifen sensitivity by the combination of IPA-3 and EBP1 overexpression are currently unknown. For example, we found that although EBP1 (both mutant and wild-type) as expected reduced levels of ErbB2 in LTLT-Ca cells, IPA-3 was able to further reduce ErbB2 levels only in LTLT-Ca-EBP1 transfectants. ErbB2 levels were decreased in both wild-type and mutant EBP1-overexpressing cells, but tamoxifen sensitivity was increased in combination with IPA only in wild-type transfectants. This finding would suggest that EBP1-induced changes in expression of additional proteins may have a role in the induction of tamoxifen sensitivity by IPA. For example, EBP1 decreases activity of the E2F1-regulated cell cycle gene Cyclin D1, which has a role in tamoxifen resistance (Xia et al, 2001a; Akinmade et al, 2008). P21-activated kinase 1 is also an important regulator of Cyclin D1 expression (Balasenthil et al, 2004). It is possible that any reduction of PAK1 phosphorylation of endogenous EBP1 in the T261A mutant transfected cells is insufficient to alter Cyclin D1 levels and subsequently tamoxifen resistance, but adequate for altering ErbB2 protein levels. 
In summary, we have found that PAK1-EBP1 interactions can increase tamoxifen sensitivity of hormone resistant breast cancer cells in part by EBP1's regulation of ErbB2 levels. An EBP1 phospho mimetic for a PAK1 site increased ErbB2 protein levels. IPA-3, a highly specific PAK1 inhibitor, abrogated tamoxifeninduced growth of hormone resistant cells and sensitised hormone resistant cells to tamoxifen in the presence of EBP1 overexpression, accompanied by decreased ErbB2 expression. These studies suggest that strategies that increase the pool of functional EBP1 and decrease PAK1 activity may affect hormone resistant cell growth. Our results also suggest that PAK1 directed therapies may be more effective in patients that express high levels of EBP1.

\section{ACKNOWLEDGEMENTS}

This work was supported by NIH grants R01 CA76047, RC1 CA145066-01, DOD W81XWH-08-1-0560 (to AWH) and R01 GM083025 (to JRP).

\section{REFERENCES}

Ahn JY, Liu X, Liu Z, Pereira L, Cheng D, Peng J, Wade PA, Hamburger AW, Ye K (2006) Nuclear Akt associates with PKC-phosphorylated Ebp1, preventing DNA fragmentation by inhibition of caspase-activated DNase. EMBO J 25: 2083-2095.

Akinmade D, Talukder AH, Zhang Y, Luo WM, Kumar R, Hamburger AW (2008) Phosphorylation of the ErbB3 binding protein Ebp1 by p21activated kinase 1 in breast cancer cells. Br J Cancer 98: 1132-1140.

Balasenthil S, Sahin AA, Barnes CJ, Wang RA, Pestell RG, Vadlamudi RK, Kumar $\mathrm{R}$ (2004) p21-activated kinase-1 signaling mediates cyclin D1 expression in mammary epithelial and cancer cells. J Biol Chem 279: 1422-1428.

Bostner J, Ahnstrom WM, Fornander T, Skoog L, Nordenskjold B, Stal O (2007) Amplification of CCND1 and PAK1 as predictors of recurrence and tamoxifen resistance in postmenopausal breast cancer. Oncogene 26 6997-7005.

Bostner J, Skoog L, Fornander T, Nordenskjold B, Stal O (2010) Estrogen receptor-alpha phosphorylation at serine 305, nuclear p21-activated kinase 1 expression, and response to tamoxifen in postmenopausal breast cancer. Clin Cancer Res 16: 1624-1633.

Clarke R, Leonessa F, Welch JN, Skaar TC (2001) Cellular and molecular pharmacology of antiestrogen action and resistance. Pharmacol Rev 53: 25-71.

Deacon SW, Beeser A, Fukui JA, Rennefahrt UE, Myers C, Chernoff J, Peterson JR (2008) An isoform-selective, small-molecule inhibitor targets the autoregulatory mechanism of p21-activated kinase. Chem Biol 15: 322-331.

Dummler B, Ohshiro K, Kumar R, Field J (2009) Pak protein kinases and their role in cancer. Cancer Metastasis Rev 28: 51-63.

Holm C, Rayala S, Jirstrom K, Stal O, Kumar R, Landberg G (2006) Association between Pak1 expression and subcellular localization and tamoxifen resistance in breast cancer patients. J Natl Cancer Inst 98: 671-680.

Hynes NE, Lane HA (2005) ERBB receptors and cancer: the complexity of targeted inhibitors. Nat Rev Cancer 5: 341-354.

Jelovac D, Sabnis G, Long BJ, Macedo L, Goloubeva OG, Brodie AM (2005) Activation of mitogen-activated protein kinase in xenografts and cells during prolonged treatment with aromatase inhibitor letrozole. Cancer Res 65: 5380-5389.

Kok M, Zwart W, Holm C, Fles R, Hauptmann M, Van't Veer LJ, Wessels LF, Neefjes J, Stal O, Linn SC, Landberg G, Michalides R (2011) PKA-induced phosphorylation of ERalpha at serine 305 and high PAK1 levels is associated with sensitivity to tamoxifen in ER-positive breast cancer. Breast Cancer Res Treat 125: 1-12.

Kurokawa H, Lenferink AE, Simpson JF, Pisacane PI, Sliwkowski MX, Forbes JT, Arteaga CL (2000) Inhibition of HER2/neu (erbB-2) and mitogen-activated protein kinases enhances tamoxifen action against HER2-overexpressing, tamoxifen-resistant breast cancer cells. Cancer Res 60: 5887-5894.

Lessor TJ, Yoo JY, Xia X, Woodford N, Hamburger AW (2000) Ectopic expression of the ErbB-3 binding protein ebp1 inhibits growth and induces differentiation of human breast cancer cell lines. J Cell Physiol 183: 321-329.
Liu Z, Ahn JY, Liu X, Ye K (2006) Ebp1 isoforms distinctively regulate cell survival and differentiation. Proc Natl Acad Sci USA 103: 10917-10922.

Lu Y, Zhou H, Chen W, Zhang Y, Hamburger AW (2011) The ErbB3 binding protein EBP1 regulates ErbB2 protein levels and tamoxifen sensitivity in breast cancer cells. Breast Cancer Res Treat 126: 27-36.

Molli PR, Li DQ, Murray BW, Rayala SK, Kumar R (2009) PAK signaling in oncogenesis. Oncogene 28: 2545-2555.

Ong CC, Jubb AM, Haverty PM, Zhou W, Tran V, Truong T, Turley H, O’Brien T, Vucic D, Harris AL, Belvin M, Friedman LS, Blackwood EM, Koeppen H, Hoeflich KP (2011) Targeting p21-activated kinase 1 (PAK1) to induce apoptosis of tumor cells. Proc Natl Acad Sci USA 108: 7177-7182.

Ou K, Kesuma D, Ganesan K, Yu K, Soon SY, Lee SY, Goh XP, Hooi M, Chen W, Jikuya H, Ichikawa T, Kuyama H, Matsuo E, Nishimura O, Tan P (2006) Quantitative profiling of drug-associated proteomic alterations by combined 2-nitrobenzenesulfenyl chloride (NBS) isotope labeling and 2DE/MS identification. J Proteome Res 5: 2194-2206.

Piccart-Gebhart MJ, Procter M, Leyland-Jones B, Goldhirsch A, Untch M, Smith I, Gianni L, Baselga J, Bell R, Jackisch C, Cameron D, Dowsett M, Barrios CH, Steger G, Huang CS, Andersson M, Inbar M, Lichinitser M, Lang I, Nitz U, Iwata H, Thomssen C, Lohrisch C, Suter TM, Ruschoff J, Suto T, Greatorex V, Ward C, Straehle C, McFadden E, Dolci MS, Gelber RD (2005) Trastuzumab after adjuvant chemotherapy in HER2-positive breast cancer. $N$ Engl J Med 353: 1659-1672.

Rayala SK, Talukder AH, Balasenthil S, Tharakan R, Barnes CJ, Wang RA, Aldaz M, Khan S, Kumar R (2006) P21-activated kinase 1 regulation of estrogen receptor-alpha activation involves serine 305 activation linked with serine 118 phosphorylation. Cancer Res 66: 1694-1701.

Sabnis G, Schayowitz A, Goloubeva O, Macedo L, Brodie A (2009) Trastuzumab reverses letrozole resistance and amplifies the sensitivity of breast cancer cells to estrogen. Cancer Res 69: 1416-1428.

Shou J, Massarweh S, Osborne CK, Wakeling AE, Ali S, Weiss H, Schiff R (2004) Mechanisms of tamoxifen resistance: increased estrogen receptorHER2/neu cross-talk in ER/HER2-positive breast cancer. J Natl Cancer Inst 96(12): 926-935.

Shrestha Y, Schafer EJ, Boehm JS, Thomas SR, He F, Du J, Wang S, Barretina J, Weir BA, Zhao JJ, Polyak K, Golub TR, Beroukhim R, Hahn WC (2011) PAK1 is a breast cancer oncogene that coordinately activates MAPK and MET signaling. Oncogene.

Squatrito M, Mancino M, Sala L, Draetta GF (2006) Ebp1 is a dsRNA-binding protein associated with ribosomes that modulates eIF2alpha phosphorylation. Biochem Biophys Res Commun 344: 859-868.

Vadlamudi RK, Adam L, Wang RA, Mandal M, Nguyen D, Sahin A, Chernoff J, Hung MC, Kumar R (2000) Regulatable expression of p21-activated kinase-1 promotes anchorage-independent growth and abnormal organization of mitotic spindles in human epithelial breast cancer cells. J Biol Chem 275: 36238-36244.

Viaud J, Peterson JR (2009) An allosteric kinase inhibitor binds the p21-activated kinase autoregulatory domain covalently. Mol Cancer Ther 8: 2559-2565.

Xia X, Cheng A, Lessor T, Zhang Y, Hamburger AW (2001a) Ebp1, an ErbB-3 binding protein, interacts with $\mathrm{Rb}$ and affects $\mathrm{Rb}$ transcriptional regulation. J Cell Physiol 187: 209-217.

Xia X, Lessor TJ, Zhang Y, Woodford N, Hamburger AW (2001b) Analysis of the expression pattern of Ebp1, an ErbB-3-binding protein. Biochem Biophys Res Commun 289: 240-244.

Yoo JY, Wang XW, Rishi AK, Lessor T, Xia XM, Gustafson TA, Hamburger AW (2000) Interaction of the PA2G4 (EBP1) protein with ErbB-3 and regulation of this binding by heregulin. Br J Cancer 82: 683-690.

Zang M, Hayne C, Luo Z (2002) Interaction between active Pak1 and Raf-1 is necessary for phosphorylation and activation of Raf-1. J Biol Chem 277: 4395-4405.

Zhang Y, Akinmade D, Hamburger AW (2008) Inhibition of heregulin mediated MCF-7 breast cancer cell growth by the ErbB3 binding protein EBP1. Cancer Lett 265: 298-306.

This work is published under the standard license to publish agreement. After 12 months the work will become freely available and the license terms will switch to a Creative Commons AttributionNonCommercial-Share Alike 3.0 Unported License. 